\title{
Tangence
}

\section{Écrire en Ontario français}

\section{Gabrielle Poulin}

Numéro 40, mai 1993

Régionalismes littéraires de la francophonie

URI : https://id.erudit.org/iderudit/025770ar

DOI : https://doi.org/10.7202/025770ar

Aller au sommaire du numéro

Éditeur(s)

Tangence

ISSN

0226-9554 (imprimé)

1710-0305 (numérique)

Découvrir la revue

Citer cet article

Poulin, G. (1993). Écrire en Ontario français. Tangence, (40), 117-130.

https://doi.org/10.7202/025770ar d'utilisation que vous pouvez consulter en ligne.

https://apropos.erudit.org/fr/usagers/politique-dutilisation/ 


\section{CONFÉRENCE}

\section{Écrire en Ontario français *}

\section{Gabrielle Poulin}

À peine avais-je accepté de participer à ce colloque et proposé le titre de ma communication que je me suis sentie piégée. Comment avais-je pu me laisser attirer, et les écrivains francophones de l'Ontario avec moi, dans le miroir aux alouettes d'un colloque dont le titre, «Écriture et région", présente une telle ambiguitée? Il faut beaucoup de courage ou d'innocence pour oser juxtaposer ainsi deux réalités sinon antagonistes du moins contradictoires. L'Écriture, la grande, celle dont la majuscule tient lieu d'épithète, n'est-elle pas, et ne doit-elle pas demeurer, l'apanage des métropoles? $\mathrm{Si}$, en région, un écrivain parvenait, malgré tout, à écrire des roman et des poèmes et même à les faire publier, ces livres ne seraient-ils pas confinés au territoire de ladite région, voués, comme les diverses activités culturelles de ce territoire, à un destin régional? Que peut-il sortir de bon d'une région?

$\mathrm{Au}$ caractère paradoxal du thème proposé s'ajoute l'incongruité de la participation d'une invitée venue de l'Ontario français. Comme si tout à coup ce spectre de pays avait acquis le droit à des frontières qui l'élèvent au statut modeste, mais rassurant, de région. Qui dit région dit métropole. À quelle métropole, me suis-je demandé, aurait-on décidé, à l'insu des FrancoOntariens, de rattacher l'Ontario français?

* Texte prononcé dans le cadre du colloque "Écriture et région", organisé conjointement par la Corporation du camp littéraire Félix et le Département des lettres de l'UQAR, les $1^{\text {er }}$ et 2 octobre 1992, à Sainte-Luce-sur-mer. 
118

Voilà le dilemme auquel j'ai dû faire face, le piège qu'on a tendu, mine de rien, aux écrivains francophones de l'Ontario. Pour pouvoir traiter du sujet convenu, il me fallait, de toute urgence, rendre ce piège inopérant. J'ai dû me résigner à séparer les deux termes de l'équation et tenter d'établir entre eux une distance rassurante. Aussi, plutôt que de les considérer d'une façon globale, ai-je choisi de les examiner l'un après l'autre de façon à échapper aux illusions et aux dangers qui guettent tout discours paradoxal.

Mais réfléchir à haute voix, dans un premier temps, sur un thème aussi général que celui de l'écriture, en présence de tant de gens à qui l'écriture a déjà révélé bien des secrets et, dans un second temps, sur un thème au contenu aussi particulier et aussi polymorphe que celui de région, n'est-ce pas s'aventurer dans l'espace mouvant des lieux communs avec le risque de s'y complaire et de s'y enliser? Si l'on a choisi d'inviter ici des romanciers et des poètes plutôt que des théoriciens de la littérature, c'est, sans doute, que l'on souhaitait entendre les participants parler, non pas de théorie, mais de leurs propres pratiques de l'écriture. Aux prises tous les jours avec des problèmes de temps, d'espace, d'images et de mots, de quoi d'autre le romancier ou le poète peut-il parler, s'il veut échapper à la stérilité des discours abstraits? L'on comprendra mieux dès lors pourquoi j'ai choisi d'écrire ce texte comme un récit et comme si je plongeais en pleine fiction. Pour éviter la condamnation qui, depuis la célèbre affirmation de Pascal, pèse sur tout discours au *je *, j'en appelle à l'impunité que garantit Kundera au *je " narrateur en reconnaissant à ce «je " l'identité d'un * ego expérimental .

Le "je" sous lequel je compte m'abriter pendant les minutes qui me sont allouées aurait fait tard l'expérience de l'écriture romanesque et plus tard encore celle de la poésie. De l'enseignement, ce «je" serait passé presque naturellement au métier de critique littéraire qu'il aurait exercé avec enthousisame et passion pendant plusieurs années. Mais à mesure qu'il se serait approché de l'univers des romanciers, il aurait senti grandir en lui un attrait mêlé de peur, une sorte de vertige des profondeurs. Il aurait eu envie de renoncer à tous les supports dont s'assure le critique et, s'abandonnant à l'euphorie d'une totale liberté, de plonger dans le vide. C'est ici que commence mon récit, ici que le "je" narrateur se croit autorisé à prendre forme du *je * expérimental. 


\section{Les personnages et leurs régions ou les fugues romanesques}

Un jour, à Québec, dans la salle à manger d'un motel où étaient réunis pour un colloque des professeurs de littérature fort expérimentés, surgit un personnage qui me parut aussi inattendu et aussi coloré que peut l'être un cardinal atterri au milieu du peuple gris et bavard des moineaux. Toutes les tables étaient occupées. Nous invitâmes le nouveau venu à s'asseoir à la nôtre. Il me fut présenté. J'appris avec une sorte de ravissement que le cardinal s'appelait Yves Thériault. J'eus l'intuition que ce colloque, enfin!, prenait un sens, que j'en sortirais métamorphosée, à la condition toutefois que j'ose poser au grand romancier une question, la seule, dont la réponse, j'en étais sûre, pouvait changer ma vie. Yves Thériault parlait beaucoup, sur toutes sortes de sujets, et avait fort bon appétit. J'écoutais, je picorais, à l'affût d'une seconde de silence. Elle vint. En toute hâte, je jetai ma question sur la table: "Yves Thériault, comment est-ce qu'on fait pour écrire un roman?" Le romancier, qui n'avait rien d'un théoricien, déposa sa fourchette sur la table. La réponse sortit directe, claire et concise: «Ma pauv' p'tite fille, c'est ben facile! T'inventes un personnage, pis tu le laisses aller." Il reprit sa fourchette et parla d'autre chose.

Dès le lundi matin, de retour sur l'autre rive de l'Ouataouais, - du mauvais côté " comme aurait dit Jacques Ferron, la pauv' p'tite fille écarta le monceau de livre à recenser, installa devant elle une pile de feuilles blanches, se débrancha de tous les appareils qu'elle avait considérés jusque-là indispensables et se laissa couler. "T'inventes un personnage, pis tu le laisses aller." Le personnage se fit attendre... longtemps. Quand il apparut enfin, je m'apprêtai à le suivre avec docilité et émerveillement là où il lui plairait de me conduire. Ou de me ramener.

Yves Thériault, que ses personnages ont fait beaucoup voyager, ne m'avait pas donné de théories sur le choix des lieux qui attirent les personnages du roman, les retiennent et les nourrissent. Du moment qu'un personnage vit, avais-je cru comprendre, il faut savoir faire confiance à son sens de l'orientation.

C'est du haut de sa galerie, d'où elle aperçoit tout le village et la petite route dépouillée et lointaine, que j'avais contemplée et interrogée si souvent dans mon enfance, que ma chère vieille Anna, mon premier personnage, la mère de la Rachel de Cogne la caboche ${ }^{1}$, me fit signe. Bien qu'il ne soit pas nommé dans le 
120

roman, ce village, c'est celui-là même où je suis née et où j'ai passé les cinq premières années de mon existence: Saint-Prosper; la région, c'est la Beauce, le pays de mes grands-parents maternels et paternels. Le jardin, le verger, la maison d'Anna, c'était l'espace des contes, "le bon côté des choses", celui où l'on n'a pas du tout envie d'écrire tant le merveilleux est à portée de la main, à toute heure du jour et de la nuit. Et j'ai compris plus tard, beaucoup plus tard, comme la Tinamer de L'amélanchier ${ }^{2}$, que l'on n'écrit jamais que de l'autre côté; que pour écrire, et cela bien des écrivains l'ont expérimenté et l'on dit avant moi, de Gérard de Nerval à James Joyce, de Gabrielle Roy à Anne Hébert, à Marie-Claire Blais, il faut avoir été déporté ou s'être éloigné de son plein gré de tout espace où l'on a cru être heureux. Pour qu'un espace puisse trouver sa place dans l'univers romanesque, il faut, en quelque sorte, qu'il nous ait été interdit.

Mais, ainsi que l'écrit Ferron à la fin de son beau conte, Tinamer, après avoir réintégré un instant le paradis déjà perdu, " ne pouvai[t] rester ainsi, retournée en arrière, quand le fil de la vie, déjà tendu, se raidissait à [lui] en faire mal pour [la] ramener en avant " 3 . À la porte du jardin perdu, Léon de Portanqueu a posté son petit ami, le jeune aveugle, le remplaçant de sa propre fille: "Tinamer, Tinamer, voici l'ange qui montera la garde." Non seulement la porte du pays de l'enfance, mais la porte de tous les lieux où a vécu le futur écrivain, même ceux où il a cru être malheureux, sont ainsi gardés dans sa mémoire, protégés des désastres écologiques, de la cupidité des spéculateurs, de la force dévastatrice et assimilatrice des modes. Quand il s'en éloigne, le futur romancier ne devrait pas s'inquiêter: son êloignement même devient pour ces lieux la condition de leur invulnérabilité et de leur survie.

Sans aller jusqu'à prétendre, comme l'auteur latin, que "où l'on est bien, là est la patrie", je peux affirmer que si je me suis toujours attachée aux lieux, aux régions où il m'a été donné d'habiter, j'ai toujours senti également que le fil tendu de la vie me tirait en avant. Je n'ai jamais pu résister longtemps à la ten-

1 Édition révisée, Montréal, VLB éditeur, coll. * Courant *, 1990, 298 p.

2 Jacques Ferron, L'amélanchier, récit, préface de Gabrielle Roy, édition préparée par Pierre Cantin, Marie Ferron et Paul Lewis, Montréal, VLB éditeur, coll. - Courant, 1986, 207 p.

Ibid., p. 154. 
sion. Aussi, chaque fois que je commence un roman, aussitôt inventé le personnage, que $j$ 'ai reçu le conseil inoubliable de laisser aller, puis-je me fier à l'appel de l'ange aveugle qui tient dans ses mains la clef de tous mes jardins.

Le jardin vers lequel se dirige la Marie-Françoise de Un cri trop grand ${ }^{4}$, c'est le jardin intact qui descendait vers la rivière, la fabuleuse Chaudière, en arrière de la maison de mes grandsparents paternels, à Saint-Martin. Peu importe que, un jour bien réel, la maison ait été détruite par le feu; celle sur laquelle veille mon ange aveugle est à l'épreuve de toutes sortes de cataclysmes.

À Saint-Vincent-de-Paul, l'ancien village du nord de Montréal, où la Crise a forcé mes parents à s'exiler quand j'avais cinq ans, mes personnages et moi sommes devenus propriétaires de trois maisons dont mes parents n'ont jamais été que locataires. Isabelle $^{5}$ a fait de l'une d'elles, à deux pas de la rivière des Prairies, la gardienne de ses mensonges. Les deux autres, l'une sur le bouelvard Lévesque, l'autre sur la rue Desnoyers, sont toujours inoccupées, dans l'attente d'un hôte encore à venir.

Sur le bord de la rivière des Prairies, voisine de la maison que nous habitions quand j'avais huit ans, se dressait une maison de pierre, inaccessible, dont nous ne voyions jamais les occupants. Cette maison, dans laquelle je rêvais de pénétrer, me fascinait. Certains jours, je me disais que c'était un cloître, d'autres jours, un orphelinat ou une crèche: de ces sortes d'institutions, dont je ne connaissais encore que le nom mystérieux découvert dans les livres. Il y a quelques années, au cours d'un voyage dans le pays de Kamouraska, auquel des circonstances heureuses de ma vie m'ont liée, j'avais entrevu un torrent dont avait un urgent besoin le personnage en pleine gestation de Florence, la femme de l'aubergiste de La couronne d'oubli ${ }^{6}$. Ce fut un jeu d'enfant pour moi que d'ordonner sur-le-champ le déménagement dans le Bas-du-Fleuve de la maison de pierre de Saint-Vincent-de-Paul qui était demeurée une énigme de mon enfance. À la suite de Florence, j'ai réussi enfin à pénétrer dans cette maison troublante. C'est encore par Florence que j'ai pu comprendre à quoi tenait l'ambiguité de l'ancien couvent métamorphosé en auberge. Con-

\footnotetext{
4 Montréal, Bellarmin, 1980, 335 p.

5 Les mensonges d'Isabelle, Montréal, Québec/Amérique, 1983, $210 \mathrm{p}$.

6 Sudbury, Prise de Parole, 1990, 178 p.
} 
trairement au cinéaste, qui bâtit sur place des reproductions factices des bâtiments qu'il ne peut transporter, le romancier a besoin de matériaux solides. Ses personnages ne jouent pas; les murs ni les paysages ne leur servent de décor. Ils réclament le corps et l'âme entière des lieux qui ne s'ouvrent que sur leur commandement. Une fois le roman terminé, ces lieux ne pourront plus être détruits, comme après un tournage, parce que les personnages refuseront toujours de les quitter. C'est seulement entre les murs où ils ont choisi de s'installer et de vivre leur destin qu'ils ont pu, en même temps que le romancier, et qu'ils pourront, avec le lecteur, épeler leur identité et leur liberté.

Ce ne sont là que quelques exemples de l'attrait que continuent d'exercer sur l'imaginaire les lieux dont, un jour, le romancier a dû se séparer. Le poète Luis Mizón a écrit: "Chaque naufrage nous a laissé quelque chose * et s'il n'a rien laissé: "des mots [qui] brillent dans les filets ${ }^{7}$. Nos épaves et nos mots sont le matériau dont les personnages de roman construisent leurs demeures.

Je m'en voudrais de ne pas parler de la Métropole dans laquelle j'ai vécu les années de mon adolescence inquiète et les longues et sages années de ma jeunesse trop austère. Ceux qui connaissent Rachel peuvent sans doute imaginer que, des fenêtres du couvent sur la montagne, dont l'obligation de la modestie des yeux et du recueillement aurait dû la tenir à l'écart, la jeune religieuse ne pouvait voir de la ville à ses pieds que des signaux encore indéchiffrables; dans son petit calepin noir, elle ne pouvait revenir hanter que le pays de l'enfance, "le bon côté des choses".

Tinamer! Tinamer! [Rachel! Rachel! lui aurait dit Jacques Ferronl, est-ce une si grande conquête que de devenir sa captive et géolière, l'une à l'autre enlacée dans un combat à n'en plus finir que tu ne sois devenue la tombe des deux? Est-ce même un avantage que d'avoir vingt ans, d'être belle et de ne plus se voir que sous de fausses représentations comme le Narcisse du miroir, de ne plus se voir que de l'extérieur, comme une autre parmi les autres, comme une quelconque catin de soi-même, alors qu'on reste seule, parmi les autres, unique et exposée sur la colline de son moi crucifiant? ${ }^{8}$

7 Luis Mizón, Jardin de ruines, traduit de l'espagnol par Jacques Ancet, Obsidiane, 1992, p. 35.

8 Jacques Ferron, op. cit., p. 153. 
Quand la religieuse quitte le couvent de la montagne, elle se demande si Rachel aura envie un jour de "revêtir à nouveau la livrée noire du fantôme de sœur Anna-des-Anges pour revenir hanter ces ruines "9. C'est dix ans après avoir quitté la métropole que j'ai pu suivre Rachel dans les corridors du cloître où elle a tenu à m'entraîner pour me dicter Cogne la caboche.

\section{Le romancier et sa région ou la force centripète de l'imaginaire}

Est-ce à dire que le souvenir ou la nostalgie des lieux que le romancier a quittés empêche à jamais ses personnages de s'établir dans l'habitat actuel, métropole ou région, celui qui est le lieu physique de l'écriture? J'ai écrit tous mes romans à Ottawa, dans une maison située tout près de la rivière des Outaouais, qui, comme chacun le sait, va se jeter dans le fleuve Saint-Laurent, en amont de la Métropole. Claire Martin a confié un jour à Bernard Pivot qu'elle avait écrit tous ses livres à Ottawa parce qu'il n'y avait là rien d'autre à faire. Je retiens de cette boutade que, quel que soit le lieu, métropole ou région, qu'habite le romancier, la seule occupation qui compte pour lui et qui fait paraitre ses autres activités accessoires, sinon dérisoires, c'est l'écriture. Il ne faut pas imaginer pour cela que dans la banlieue d'Ottawa, où j'habite depuis plus de vingt ans et où j'écris, j'ai toujours les yeux tournés vers l'autre côté de la rivière et que je hante constamment les régions, demeurées très chères, qui me sont désormais inaccessibles dans la réalité.

Si mes personnages m'ont toujours entraînée vers cet ailleurs, toujours ils m'ont aussi sûrement ramenée chez moi. C'est l'écriture qui m'a permis de m'enraciner en Ontario français, qui m'a fait découvrir la vie et la fécondité d'un espace qui, de loin, peut paraître, à d'autres, étranger et stérile. Dans Donner à voir, Paul Éluard a écrit: "Tout est comparable à tout, tout trouve son écho, sa raison, sa ressemblance, son opposition, son devenir partout. Et ce devenir est infini. " 10

En écrivant La couronne d'oubli, j'ai pris conscience que, à l'exemple des géants des contes de l'enfance, le romancier exerce un pouvoir qui fait échec aux prétentions des géographes et des

9 Cogne la caboche, p. 271.

10 Dans Eutres complètes, Paris, Gallimard, coll. a La Pléiade ^, 1968, p. 97. 
cartographes. Non seulement peut-il déménager les maisons sur lesquelles ses personnages ont jeté leur dévolu et les installer à des milles et des milles de distance de leur emplacement originel, mais il peut même déplacer les montagnes et les rivières, si le sort de ses personnages le réclame. L'écriture est la grande réconciliatrice: elle se nourrit de réalités en apparence contradictoires et irréconciliables; de leurs contrastes et de leur rapprochement inattendu et insolite, elle tire le souffle d'une nouvelle vie. Les personnages du roman se jouent des distances; ils font fi également des frontières physiques et psychologiques. Les eaux du ruisseau des Abénaquis à Saint-Prosper, celles de la rivière Chaudière, de la rivière des Prairies, de la rivière du Nord et même celles du torrent entrevu dans Kamouraska, se sont mêlées aux eaux de l'Outaouais pour alimenter la rivière omniprésente dans mes romans. Dans le verger de Cogne la cabocbe, la cédrière des Mensonges d'Isabelle et le bois de La couronne d'oubli, il y a des arbres dont les racines s'enfoncent dans le sous-sol de l'Ontario. Quant aux personnages eux-mêmes, si leurs désirs, leurs rêves, leurs mensonges, leurs regrets, leur envie de vivre étaient déjà en germe dans les rencontres et les apprentissages du pays de l'enfance, comme au jardin de Tinamer, dans le petit bois où chante le loriot et fleurit l'amélanchier, c'est la rencontre et le contact d'êtres réels de l'Ontario français, des êtres à la fois semblables et différents, qui leur a permis de découvrir leur véritable identité et de faire, dans l'univers des mots, l'apprentissage et la conquête de leur liberté.

Non, ce n'est pas la nostalgie ni le désœuvrement, comme le suggère Claire Martin, qui, à Ottawa ou ailleurs, jettent l'écrivain en puissance dans l'aventure de l'écriture. Le romancier que les circonstances ont éloigné des lieux de son origine, mis à l'écart de la vie exaltante et dévorante d'une métropole où cherchent à se concentrer les rouages de l'institution littéraire, doit pouvoir trouver une terre solide et nutritive dans laquelle il puisse s'enraciner pour que ses personnages prennent forme et que ses romans soient viables. S'il arrive qu'un écrivain quitte la région où il a commencé à écrire pour gagner la métropole, et cela arrive, ce n'est pas nécessairement pour enrichir la vie privée et secrète de ses personnages qu'il le fait, mais plutôt pour le succès de leur vie publique. La Sagouine était déjà bien vivante quand elle est arrivée à Montréal; Luzina aurait fort bien pu demeurer féconde au Manitoba et je ne suis pas sûre que "l'Homme invisible" ait quitté le pays des mines pour suivre Patrice Desbiens dans son exil. 
Quand il écrit, même le romancier qui vit dans la métropole essaie de se tenir à l'écart de l'institution despotique, des chapelles ombrageuses, des modes insidieuses et nivelantes, des médias tapageurs voire des colloques. Ça ne doit pas être tous les jours facile. Le silence institutionnel et médiatique qui entoure l'acte créateur du romancier qui vit en région le protège, lui et son univers, des dangers de la contamination. Milan Kundera écrit:

Le roman ne peut vivre en paix avec l'esprit de notre temps: s'il veut encore continuer à découvrir ce qui n'est pas découvert, s'il veut encore "progresser* en tant que roman, il ne peut le faire que contre le progrès du monde. ${ }^{11}$

George Steiner renchérit: "Je crois que la création du poète [...] est une contre-création. " 12

Le romancier et le poète qui demeurent loin de la métropole, je serais surprise qu'ils considèrent la distance comme un facteur d'appauvrissement de leur imaginaire. Pour être moins trépidante que la vie métropolitaine, la vie en région est-elle moins intense? La précarité de toute vie humaine, la puissance et la fragilité des passions, serait-il possible qu'elles soient moins ressenties en région? Quant à la recherche de sa propre identité et à la poursuite de la liberté, existe-t-il quelque espace qui leur soit interdit?

-Le romancier n'est ni historien ni prophète: il est, encore selon l'affirmation de Kundera, explorateur de l'existence." ${ }^{13} \mathrm{Ce}$ métier et cet art échappent aux diktats des puissances extérieures; ma propre expérience m'enseigne que le romancier qui réussit à se tenir loin, physiquement et spirituellement, des lieux où l'institution littéraire exerce son pouvoir est moins distrait de ce qui est le cœur de l'acte d'écrire.

Les œuvres de l'écrivain qui écrit en région et qui publie chez des éditeurs régionaux ne font pas partie du défilé de mode qu'amène avec elle dans la métropole chaque nouvelle saison littéraire. Leurs débuts sont modestes. Le travail de l'écrivain est à peine interrompu pendant les semaines qui suivent la parution de son livre. C'est un grand avantage que la tranquillité d'esprit et le silence pour celui qui *n'est pas fasciné par sa voix, mais par une

11 L'art du roman, Paris, Gallimard, 1986, p. 35.

12. Réelles présences. Les arts du sens, traduit par Michel R. de Pauw, Paris, Gallimard, coll. * NRF essais *, 1992, p. 244.

13 Milan Kundera, op. cit., p. 63. 
126

forme qu'il poursuit, [car] seules les formes qui répondent aux exigences de son rêve font partie de son œuvre "14.

\section{La vie lente et tenace des livres en Ontario français}

Est-ce à dire que le roman ou le recueil de poèmes de l'écrivain qui vit en région est condamné à l'oubli le jour même de sa publication? Au contraire, en région, les livres ne font pas encore partie des biens de consommation. Les maisons d'édition ne sont pas entrées dans l'ère industrielle. Les auteurs ne se marchent pas sur les pieds et les lecteurs ne trouvent pas une librairie à tous les coins de rue. Il y a même des villes à forte population francophone en Ontario qui n'ont pas de librairie française. Ainsi, les Éditions Prise de parole de Sudbury ne peuvent compter sur les services d'aucune librairie française dans cette ville importante de la francophonie ontarienne. Pourtant, en région, les lecteurs lisent leurs auteurs. La maison Prise de parole a deux cent cinquante abonnés qui reçoivent, dès leur parution, tous les livres publiés. À Hearst non plus, le foyer du Nordir, il n'y a pas de grande librairie française, mais, dans le hall du Collège universitaire, dans une vitrine bien en vue, sont exposée d'une façon permanente les ouvrages anciens et récents du Nordir.

Les lancements d'ouvrages en région sont plus rares que dans la métropole; c'est pourquoi la parution d'un roman ou d'un recueil de poésie prend l'importance d'un événement littéraire et donne lieu à une fête, modeste il est vrai, presque une fête de famille, qui rassemble auteurs et lecteurs. Le livre fait ses débuts dans l'intimité et dans l'amitié. La presse locale le salue; il fait l'objet d'un compte rendu dans un périodique régional, s'il s'en trouve. Dans certains cas, rares il faut bien l'avouer, sa renommée se répandra au-delà des frontières de ladire région et le livre exotique aura droit aux minces avantages d'un marché déjà encombré qui, presque chaque semaine, fait place nette devant l'arrivée des produits frais.

En région, l'on n'oublie pas si cavalièrement ses auteurs. Quelques-unes des rares librairies françaises d'Ottawa tiennent à conserver, au long des années, au moins un exemplaire des œuvres publiées en Ontario français. Chaque année, au Salon du livre de l'Outaouais, les éditeurs de l'Ontario ont le souci d'offrir

14 Ibid., p. 180-181. 
aux lecteurs, avec les ouvrages nouveaux, les ouvrages publiés les années précédentes alors que les éditeurs de la métropole, qui profitent de la publicité de ce salon régional, oublient la plupart du temps de présenter les livres publiés chez eux par des auteurs de la région l'année précédente et remplissent leurs étagères des best-sellers anciens et nouveaux de la métropole.

En région, ce n'est que lentement que le livre rejoint le lecteur qui, dans bien des cas, doit faire des efforts pour se le procurer. Mais un livre sur lequel n'ont pas pesé les contraintes de la mode sera moins exposé à être mis au rancart comme un vêtement de l'année précédente, un livre sur lequel les dogmes des chapelles, hors desquelles il n'y a point de salut, n'ont pas exercé leur puissance réductrice, ne sera pas non plus entraîné dans la débâcle des apostasies et des hérésies.

En région, de plus en plus de chercheurs s'intéressent au patrimoine littéraire et le mettent en valeur dans des histoires littéraires, des bibliographies et des anthologies qui sont destinées, non seulement aux étudiants, mais à un public qui découvre avec étonnement et fierté la richesse de sa culture. Dans les universités et les collèges des régions, on ose mettre au programme des cours de littérature régionale.

Le tableau que je viens de brosser à grands traits peut parâ̂tre idyllique. Je l'ai fait en réfléchissant à ma propre expérience d'écrivain dans la région de l'Outaouais, "du mauvais côté des choses". Je dois l'avouer: j'ai longtemps hésité avant de confier un manuscrit à un éditeur franco-ontarien. Même si je vis et écris en Ontario depuis 1970, c'est dans la métropole que j'ai publié mes trois premiers romans ainsi que mes ouvrages de critique. En 1990, j'ai pris le risque de présenter le manuscrit de La couronne d'oubli à Denise Truax, aux Éditions Prise de parole. Je ne m'en suis jamais repentie. Pas plus qu'Hélène Brodeur qui, après avoir publié aux Éditions Quinze de Montréal les deux premiers tomes de Chroniques du Nouvel Ontario, les a réédités aux Éditions Prise de parole qu'elle avait choisies pour son troisième tome ${ }^{15}$. Après avoir publié son premier recueil de nouvelles, La chambre à mourir, qui a fort bien été accueilli par la critique, aux Éditions

15 Chroniques du Nouvel Ontario, tome 1: La quête d'Alexandre, 1985, 283 p.; tome 2: Entre l'aube et le jour, 1986, 233 p.; tome 3: Les routes incertaines, $1986,235 \mathrm{p}$. 
L'instant même de Québec ${ }^{16}$, Maurice Henrie a opté, lui aussi, pour un éditeur franco-ontarien: il fait paraître, cet automne, un second recueil de nouvelles, Le pont sur le temps ${ }^{17}$, aux Éditions Prise de parole.

J'imagine que ces livres ont connu et connaîtront dans les librairies montréalaises à court d'espace le sort des dizaines pour ne pas dire des quelque deux cents romans qui se publient chaque année, et que la plupart des libraires ne se donnent pas la peine de commander à la lointaine maison de Sudbury l'exemplaire qu'un lecteur souhaiterait obtenir. Mais, en Ontario et dans la région de l'Outaouais, il y a chaque année des lecteurs qui, grâce au Salon du livre et aux bibliothèques, parfois aux librairies, font la découverte d'auteurs franco-ontariens dont ils parlent à leurs parents, à leurs amis, à leurs étudiants. Un exemple: à Belle-Rivière, près de Windsor, la librairie Boukaliure a organisé pendant l'été, en collaboration avec le journal francophone $L e$ rempart, un concours de lecture pour les adultes et les adolescents à partir d'une liste d'ouvrages. Les lecteurs gagnants viennent d'être publiés dans deux pages du journal ${ }^{18}$. Le succès de cette initiative a convaincu les organisatrices du concours de renouveler l'expérience l'été prochain. N'est-ce pas tout ce que peut désirer un romancier, outre le plaisir d'écrire, que celui que ses romans, année après année, demeurent accessibles à de nouveaux lecteurs, pour que ses personnages continuent de vivre et de se faire des amis intimes et fidèles?

En 1991, c'est à Robert Yergeau, qui dirige les Éditions du Nordir de Hearst, que j'ai présenté le manuscrit de mon premier recueil de poésie. Je souhaite à tous les poètes de la métropole un accueil aussi personnel, une expérience aussi stimulante. J'imagine que Gaston Miron, René Bonenfant et Céline Fortin réservaient cette sorte d'accueil aux auteurs de leur maison. L'aventure inespérée de mes Petites fugues pour une saison sèche ${ }^{19} \mathrm{~m}$ 'a fait toucher du doigt à quel point le traitement industriel et commercial qu'on réserve aux livres dans les métropoles est un traitement contre nature et à quel point, en dépit des lacunes, dues à l'environnement anglophone plutôt qu'à leur

$161989,196 \mathrm{p}$.

$171992,155 \mathrm{p}$.

18 Le rempart, 16 septembre 1992, p. 3 et 7 .

19 Hearst, Le Nordir, coll. «Poésie *, 1991, 91 p. 
façon de fonctionner, des maisons comme Prise de parole et Le Nordir sont essentielles aux auteurs qui vivent en région et au public lecteur. Ces éditeurs et leurs auteurs souhaiteraient certes pouvoir profiter du marché montréalais. Celui-ci est déjà encombré par la production d'une autre métropole, la française, dont éditeurs et auteurs déplorent l'attitude arrogante et colonisatrice envers tout ce qui n'est pas de Paris. Là-bas, l'on qualifie volontiers de régionale la littérature qui naît de l'autre côté de l'Atlantique, fût-ce dans une autre métropole. Au pays de la francophonie universelle, quelque grand que l'on se considère, l'on est toujours la région d'une autre métropole.

En Ontario, il existe également un programme du ministère de la Culture et des Communications intitulé "Écrivains dans les bibliothèques publiques ". De nombreux auteurs franco-ontariens ont pu participer à ce programme qui leur a permis de rencontrer des lecteurs et d'animer des ateliers de création littéraire. Des ateliers existent aussi hors les murs. Ainsi, depuis trois ans est à l'œuvre dans la région de l'Outaouais un atelier qui regroupe quinze adultes des deux rives de l'Outaouais. Il y a quelques mois, ce groupe s'est donné une constitution et un non: "L'Atelier de création littéraire de l'Outaouais " ('ACLO). Il se réunit une fois par mois, le dimanche matin, pendant trois heures, et pas nécessairement parce qu'il n'y aurait rien d'autre à faire le dimanche à Ottawa. Les travaux de l'atelier ont donné des résultats fort encourageants: ils ont conduit, entre autres, à la préparation d'un recueil de nouvelles collectif qui paraîtra aux Éditions Prise de parole pour le Salon du livre de l'Outaouais en mars 1993, sous le titre Rendez-vous place de l'Horloge. Les participants de l'ACLO, des femmes et des hommes qui ont entre 30 et 60 ans, forment un groupe de lecteurs, de critiques et d'amis. Ils veulent écrire; ils écrivent déjà; presque tous ont entrepris la rédaction d'une œuvre de longue haleine: roman, recueil de nouvelles ou de poèmes. Ils mettent en commun leurs difficultés, échangent leurs expériences; ils se lisent les uns les autres; ils sont dans leur famille, parmi leurs amis, dans leur milieu de travail, des promoteurs de la littérature et contribuent d'une façon active à la vie littéraire de la région. Leur présence et leur enthousiasme suffiraient, si besoin en était encore, à me convaincre que, après tout, "écriture" et "région" sont bien loin d'être des termes irréconciliables. La vie littéraire en Ontario français est pleine de promesses. Sans doute aurais-je pu apporter d'autres exemples de sa vitalité et de son rayonnement. Je m'en voudrais d'oublier le rôle 
important que jouent les postes de radio régionaux pour la promotion des œuvres écrites et publiées en région et l'attention exceptionnelle dont font l'objet de la part de la chaîne française de TV Ontario les ouvrages littéraires des Franco-Ontariens.

Je crois qu'un colloque comme celui-ci permet à chacun de prendre conscience de la richesse littéraire et du dynamisme créateur de cette réalité qui s'appelle une région et de l'importance d'établir des liens entre les régions, non pas pour qu'elles se défendent mieux contre l'indifférence, l'oubli ou le mépris des métropoles, mais pour qu'elles prennent davantage conscience du caractère unique, et j'ose dire sacré, de l'écriture qu'elles continuent de protéger de l'uniformisation industrielle et des compromis que nécessitent les succès commerciaux.

Selon le vœu de George Steiner, les œuvres littéraires, comme toutes les véritables œuvres de l'art, devraient créer un

I...l contre-monde tellement entier, tellement marqué du sceau de l'artisan $[. .$.$] que ce monde frappera à la porte de notre âme$ et y entrera; et qu'à notre tour, nous lui ferons écho, nous l'abriterons dans notre souvenir, en y découvrant une résidence pour nos besoins et nos reconnaissances les plus intimes. ${ }^{20}$

C'est peut-être ce contre-monde qui cherche à atteindre Tinamer, exilée dans l'univers adulte, comme dans une métropole. Où que se trouve l'écrivain quand il écrit, c'est toujours ce contre-monde qui devrait apparaître; c'est lui, "le bon côté des choses".

20. George Steiner, op. cit., p. 244. 